\title{
Management of rhythm disorders in Duchenne muscular dystrophy
}

\author{
Jeffrey Bennett ${ }^{1}$ and Naomi Kertesz ${ }^{1}$ \\ ${ }^{1}$ Nationwide Childrens Hospital
}

August 14, 2020

\begin{abstract}
Dystrophin-deficiency results in a cardiomyopathy of variable onset and deficiency. Myocardial scarring commonly results in cardiac dysfunction, with both atrial and ventricular dysrhythmias. Heart failure, rather than arrhythmia burden, remains the strongest cardiac predictor of mortality in this patient population. Current data suggests the overall rate of sudden cardiac death in pediatric dilated cardiomyopathy is significantly lower than adults. Specifically in the Duchenne cardiomyopathy population, sudden death from an arrhythmic cause appears to be rare, even in patients with previously diagnosed arrhythmias. Despite this, recommendations for implantable cardioverter-defibrillator (ICD) placement in patients with Duchenne cardiomyopathy has traditionally been extrapolated from adult heart failure recommendations based on decreased left-ventricular ejection fraction less than 35\%. Early involvement of the cardiologist in the care for patients with dystrophin-deficient cardiomyopathy is recommended for this reason. The indications for ICD placement to prevent sudden death in patients with Duchenne cardiomyopathy are not well defined. There is little evidence to suggest placement meaningfully prolongs life in this population, and should be carefully considered in accordance with the care goals of the patient and his family.
\end{abstract}

Management of rhythm disorders in Duchenne muscular dystrophy: is sudden death a cardiac or pulmonary problem?

Jeffrey Bennett and Naomi Kertesz

Nationwide Childrens Hospital, Pediatric Cardiology

Naomi.kertesz@nationwidechildrens.org

\section{Introduction}

Dystrophinopathies in the form of Duchenne and Becker's muscular dystrophies affect 1:5000 newborn males worldwide ${ }^{1}$. As patients age, myocyte degeneration and myocyte fibrosis occur, resulting in progressive immobility and respiratory failure. While neuromuscular disease is severe in Duchenne Muscular Dystropy (DMD) and milder in Becker's Muscular Dystrophy (BMD), dystrophin deficient cardiomyopathy tends to be more unpredictable in onset. Myocardial fibrosis and arrhythmic manifestations are common and do not necessarily correlate to the degree of skeletal muscle involvement ${ }^{2}$. In fact, in cases of more mild muscular disease, cardiac dysfunction may precede symptomatic skeletal muscle involvement ${ }^{3}$. Periodic cardiac evaluation including imaging and routine surveillance of cardiac arrhythmias is recommended, and advanced imaging frequently detects myocardial fibrosis under the age of ten years old ${ }^{4}$.

\section{Mechanisms for Arrhythmias in Duchenne Cardiomyopathy}

The substrate for arrhythmias in DMD remains under investigation. Progressive myocardial fibrosis may produce scar which disrupts normal myocardial electrical coordination. Fibrosis manifests as late gadolinium enhancement (LGE) on cardiac magnetic resonance (CMR) imaging, most often involving the posterobasal subepicardial muscle. Advanced disease features LGE expanding to the inferior and lateral wall, with increasing thickness of affected myocardium ${ }^{5,6}$. Electrocardiogram (ECG) abnormalities in DMD were described 
greater than 50 years ago and in general are thought to mirror the pattern of myocardial injury ${ }^{7}$. Altered electrical activation (depolarization) results in changes to the QRS complex voltage and are commonly identified $^{8,9}$. Fragmented QRS complexes in adults represent heterogeneous myocardial activation in both ischemic and non-ischemic cardiomyopathy have been associated with arrhythmic events and sudden cardiac death $^{10}$. Specifically in the DMD population, QRS fragmentation is associated with both left ventricular systolic dysfunction and ventricular ectopy burden ${ }^{11}$.

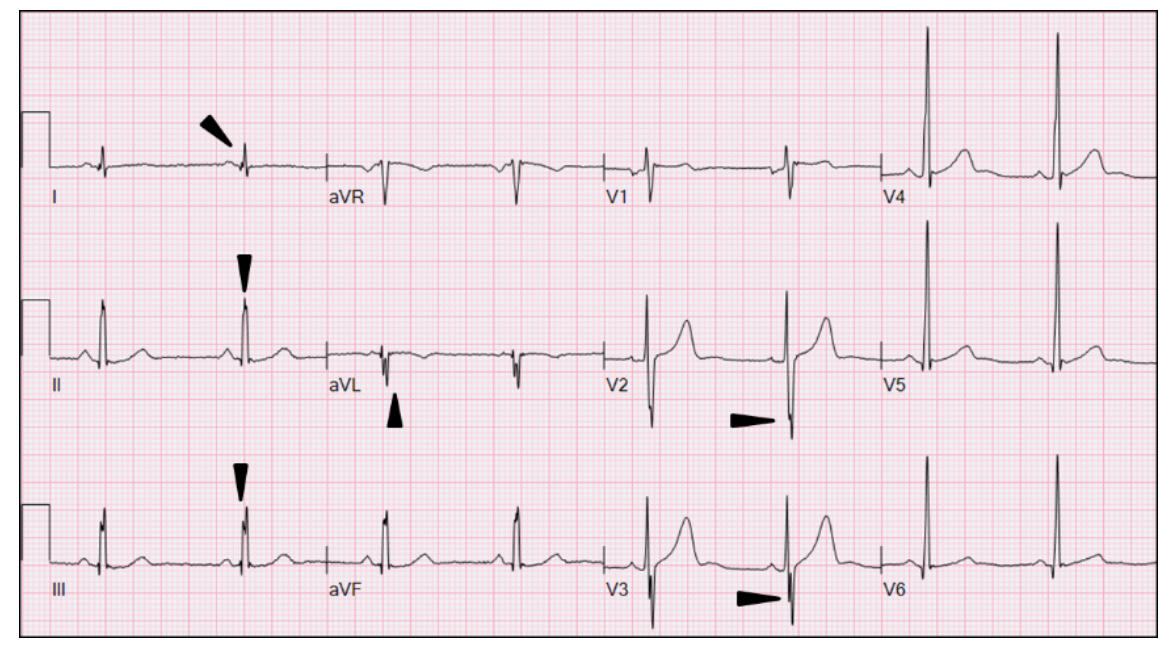

Figure 1: ECG of a patient with dystrophin-deficient cardiomyopathy. Complex QRS morphology and fragmentation reflect abnormal depolarization of myocardium.

The mechanism for arrhythmia in the dystrophin-deficient cardiomyopathy involves not just fibrosis of the myocardial tissue, but derangement of the molecular electrophysiology of conduction system cells themselves. These specialized cells are reliant on dystrophin to support organization of ion channels and promote coordinated electrical activity ${ }^{12}$. In mouse models deficient in dystrophin, misregulation of critical ion channels in specialized conduction tissue cells disturbs electrical activity, contributing to the arrhythmogenesis in the dystrophin-deficient heart ${ }^{13,14}$. On electrocardiogram, both short and long PR intervals may be observed, and complete heart block has been observed ${ }^{15}$.

\section{Genotype-Phenotype Correlation}

The dystrophin deficient cardiomyopathies, Becker's and Duchenne Muscular Dystrophy differ in both genetic alteration, and residual protein function. Within each disease however, further attempts to correlate specific genotype and phenotype have been made ${ }^{16}$. Patients with sparing of certain crucial domains within the protein, or mutations which replace a single amino acid rather than disrupting the entire protein reading frame, trend towards having milder cardiac phenotype on magnetic resonance imaging ${ }^{17}$. While mutation analysis may help to predict age of onset of cardiomyopathy in the muscular dystrophy, arrhythmia burden has not included in these studies ${ }^{18,19}$.

\section{Arrhythmias in DMD}

Despite a compelling substrate and abnormal conduction system tissue, arrhythmia burden in DMD is generally attributed to accumulated cardiac injury and systolic dysfunction. The most common finding on ambulatory monitoring of patients with DMD is sinus tachycardia. Often in response to pulmonary pathology or cardiac dysfunction, atrial arrhythmias including flutter and fibrillation may develop ${ }^{7}$. In a study of 151 patients with dystrophinopathy (Duchenne and Becker's), arrhythmias were significantly associated with decreased function, as well as independently with increased $\mathrm{age}^{20}$. In the study over 300 Holter monitor studies from Texas Children's Hospital were evaluated, and burden of supraventricular tachycardia (SVT), 
ventricular tachycardia (VT), or frequent ventricular premature contractions (VPCs) were noted. Overall, $44 \%$ of patients with DMD and 57\% of patients with BMD had arrhythmias, with clinically significant arrhythmia, either SVT or VT, occurring in $10 \%$ and $25 \%$ of those patients respectively ${ }^{20}$. Though PR interval and QRS disturbances have demonstrated the progressive impact of dystrophin deficiency on cardiac conduction, complete atrioventricular block is $\operatorname{rare}^{21,22}$. There was correlation between atrial arrhythmias and sudden cardiac death in dystrophin-deficient cardiomyopathies controlling for decreased systolic function. In fact atrial arrhythmias were present in $68 \%$ of all holter monitors in one study on 235 patients with DMD ${ }^{23}$. Non-sustained atrial tachycardia, including frequent premature atrial contractions, are more common in patients with decreased systolic function. None of the patients experienced sustained atrial tachycardia and just 5 patients $(2 \%)$ experienced ventricular tachycardia. Other rare arrhythmias including bundle branch reentry ventricular tachycardia, have been noted ${ }^{7,23}$. Atrial fibrillation and flutter, certainly common in the adult dilated cardiomyopathy population, is much rarer in children. When these occur, increased cardiac rhythm monitoring and treatment with standard antiarrhythmic therapies is recommended ${ }^{2}$.

\section{Clinically Significant Arrhythmias in Muscular Dystrophy}

Cardiorespiratory monitoring during hospitalization of muscular dystrophy patients with muscular dystrophy has allowed for some insight into causes of cardiac arrest in this population. A large retrospective study of the Pediatric Health information Database (PHIS) identified 84 cardiac arrests in 3363 patients with muscular dystrophy who were hospitalized ${ }^{24}$. An analysis of ICD-9 codes found that cardiac arrest/mortality rate was 5 fold higher (1\% vs 5\%) in patients with cardiac disease. In patients with ventricular tachycardia (VT) encoded at some point in the stay, the rate was significantly higher, with or without heart failure. Risk factors for death were VT, inotrope use, and cardiomyopathy, as well as ventilator use, reinforcing the suggestion of complex cardiopulmonary interactions at the heart of death in this population.

\section{Prevention of Sudden cardiac death}

Sudden cardiac death secondary to heart failure with reduced systolic function is well-studied in the adult population, and decreased ejection fraction $(<35 \%)$ remains an indication for primary prevention ICD placement, even in absence of documented ventricular tachyarrhythmia ${ }^{25,26}$. ICD placement has been shown quite definitively to reduce mortality in this adult population ${ }^{27}$. In the pediatric dilated cardiomyopathy population, the mechanism of death is more likely to be a heart failure death rather than a sudden arrhythmic death. This incidence of sudden death in the pediatric age group is far lower than what is found in the adult cohorts. It is therefore far more difficult to predict sudden cardiac death from decreased function alone, and far fewer primary prevention ICDs are placed in the setting of decreased LV ejection fraction without concurrent clinically significant arrhythmias. A study of 85 patients with idiopathic dilated cardiomyopathy and mean ejection fraction of $25 \%$ found just one sudden cardiac death, despite a $60 \%$ mortality rate over the course of the study ${ }^{28}$. The study, which excluded dystrophin-deficient cardiomyopathy, found no statistically significant difference in survival among patients with either atrial or ventricular arrhythmias, and the majority of deaths were from heart failure or transplantation. The Pediatric Cardiomyopathy Registry also sought to answer this question in a registry of over 3,500 infants, children and adolescents younger than 18 years old $^{29}$. Despite a high overall five year mortality rate, the 5 year sudden cardiac death rate was estimated at $3 \%$ (of 280 overall deaths), far below the $14 \%$ two-year mortality rate reported in adults without ICD implantation ${ }^{27}$. Left ventricular dilation with posterior wall thinning and early onset of clinic disease $(<14.3$ years) were significantly associated with risk of sudden cardiac death. Antiarrhythmic medication usage was associated with SCD, however detailed arrhythmias and holter data was not available.

While arrhythmias are common in advanced Duchenne cardiomyopathy, declining left ventricular systolic function remains the most powerful cardiac predictor of mortality though it does not predict whether it will be due to sudden arrhythmic death or a heart failure death ${ }^{30}$. A study of 442 holters across 235 patients found late gadolinium enhancement, left ventricular dilation and older age were all associated with decreased cardiac function. Most importantly, patients with severe decrease in systolic cardiac function (LV EF $<35 \%)$ were at higher risk for clinically significant arrhythmias. While 3 study patients died over the four year timeframe, none had cardiac death, or severe dysfunction ${ }^{23}$. 
To help mitigate mortality secondary to systolic dysfunction in Duchenne cardiomyopathy, pharmacotherapy has been generally adopted from adult consensus statements, which are frequently updated ${ }^{31,32}$. Inhibition of the renin-angiotensin system inhibition, via angiotensin converting enzyme inhibition(ACEI) or angiotensin receptor blockers (ARB) remains first line treatment, and have been demonstrated to improve LV systolic function in $\mathrm{DMD}^{33}$. Studies in the past decade have identified benefit from aldosterone inhibition with either spironolactone or eplerenone ${ }^{34-36}$, though the use of these agents is too new to demonstrate mortality benefit. Beta-adrenergic antagonists are a mainstay of treatment in adult heart failure, and when used in children with dilated cardiomyopathy may serve to decrease the burden of ventricular arrhythmias as well.

Current treatment guidelines support ICD implantation in patients with medically treated nonischemic cardiomyopathy (LVEF [?] 35\%) and mild heart failure symptoms, with recommendation of Class $\mathrm{I}^{37}$. The supportive data for this recommendation are predominantly derived from the SCH-HeFT trial which enrolled patients with a median age of 60 years and included those with ischemic cardiovascular substrate ${ }^{38}$. It is unclear if these results and documented benefit can be readily extrapolated to the much younger muscular dystrophy population ${ }^{39}$. Additional data from the Pediatric Cardiomyopathy Registry and other similar series demonstrate very low rate of sudden arrhythmic death in the pediatric population, contrasting their adult couterparts ${ }^{28,29,40}$. Consequently, it remains unclear at what age the incidence of sudden arrhythmic death increases and when ICD implantation is most beneficial. Additional considerations for ICD implantation in this population include the technical challenge difficulties brought about by body habitus including severe kyphoscoliosis, respiratory muscle weakness, and sedation-related complications ${ }^{2}$. A multi-center study recently sought to describe the use of ICDs in advanced Duchenne cardiomyopathy, reviewing 436 males with DMD. Of these, 57 patients (13\%) had severe dysfunction, and just nine underwent ICD implantation. There were two appropriate shocks and no inappropriate shocks in the study, and just 3/16 deaths in severe LV dysfunction group. Nine additional patients met ICD implantation criteria but did not receive an ICD. Four of these patients died (respiratory failure, multisystem organ failure and an embolic stroke following VAD and of unknown etiology). The authors concluded that DMD cardiomyopathy has similarities to both ischemic and non-ischemic cardiomyopathy, and that for patients with symptoms on GDMT, ICD implantation may be beneficial ${ }^{41}$. It is important to note that many of these patients in this study were not on guideline directed medical therapy. Surveys of patients with DMD and caregivers do suggest interest in a discussion about potential benefits of ICDs, should a life-prolonging benefit be demonstrated ${ }^{42}$.

\section{Conclusions}

Repeat investigations have demonstrated that sudden cardiac death in patients with dilated cardiomyopathy and severely diminished LV systolic function is far rarer in the pediatric population than in adults. Despite this, recommendations for ICD implantation in DMD are generally extrapolated from adult data into the pediatric population. Moreover, our advances in heart failure have allowed children with Duchenne cardiomyopathy to grow into adults with Duchenne cardiomyopathy, and the need for evidence-based disease-specific recommendations is great. There is limited evidence to suggest sudden death in Duchenne Muscular Dystrophy is typically from an arrhythmic cause. More likely, pathologic cardiopulmonary interactions manifest as sudden death. The inability to correlate holter findings to cardiac arrest risk make discussions about ICD placement challenging, as there is not significant evidence suggesting placement meaningfully prolongs life in these patients. The placement of an ICD is not without risk; the patient will need anesthesia which may require intubation or other respiratory support. In our institution, our practice is to discuss ICD placement only in presence of both severely decreased ejection fraction and ventricular arrhythmias. While ICDs are an effective method to prevent arrhythmic death in cardiac disease, the absolute risk of sudden cardiac arrest in the dystrophin-deficient cardiomyopathies is low, and the decision to implant an ICD must be thoughtfully discussed with the patient and his caregivers, and in accordance with their goals of care.

1. Buddhe S, Cripe L, Friedland-Little J, et al. Cardiac Management of the Patient With Duchenne Muscular Dystrophy. Pediatrics.2018;142(Suppl 2):S72-S81.

2. Birnkrant DJ, Bushby K, Bann CM, et al. Diagnosis and management of Duchenne muscular dystrophy, part 2: respiratory, cardiac, bone health, and orthopaedic management. Lancet Neurol. 2018;17(4):347-361. 
3. Melacini P, Fanin M, Danieli GA, et al. Myocardial involvement is very frequent among patients affected with subclinical Becker's muscular dystrophy. Circulation. 1996;94(12):3168-3175.

4. Hor KN, Taylor MD, Al-Khalidi HR, et al. Prevalence and distribution of late gadolinium enhancement in a large population of patients with Duchenne muscular dystrophy: effect of age and left ventricular systolic function. J Cardiovasc Magn Reson. 2013;15:107.

5. Bilchick KC, Salerno M, Plitt D, et al. Prevalence and distribution of regional scar in dysfunctional myocardial segments in Duchenne muscular dystrophy. J Cardiovasc Magn Reson. 2011;13:20.

6. Johnston TP, Cripe LH, Mah ML, et al. Young Becker Muscular Dystrophy Patients Demonstrate Fibrosis Associated With Abnormal Left Ventricular Ejection Fraction on Cardiac Magnetic Resonance Imaging. Circ Cardiovasc Imaging. 2019;12(7):e008919.

7. Rajdev A, Groh WJ. Arrhythmias in the muscular dystrophies. Card Electrophysiol Clin. 2015;7(2):303308.

8. Fitch CW, Ainger LE. The Frank vectorcardiogram and the electrocardiogram in Duchenne progressive muscular dystrophy. Circulation. 1967;35(6):1124-1140.

9. Slucka C. The electrocardiogram in Duchenne progressive muscular dystrophy. Circulation. 1968;38(5):933-940.

10. Das MK, Zipes DP. Fragmented QRS: a predictor of mortality and sudden cardiac death. Heart Rhythm. 2009;6(3 Suppl):S8-14.

11. Cho MJ, Lee JW, Lee J, Shin YB, Lee HD. Relationship Between Fragmented QRS Complexes and Cardiac Status in Duchenne Muscular Dystrophy: Multimodal Validation Using Echocardiography, Magnetic Resonance Imaging, and Holter Monitoring. Pediatr Cardiol.2017;38(5):1042-1048.

12. Gavillet B, Rougier JS, Domenighetti AA, et al. Cardiac sodium channel Nav1.5 is regulated by a multiprotein complex composed of syntrophins and dystrophin. Circ Res. 2006;99(4):407-414.

13. Albesa M, Ogrodnik J, Rougier JS, Abriel H. Regulation of the cardiac sodium channel Nav1.5 by utrophin in dystrophin-deficient mice.Cardiovasc Res. 2011;89(2):320-328.

14. Ebner J, Uhrin P, Szabo PL, et al. Reduced $\mathrm{Na}(+)$ current in Purkinje fibers explains cardiac conduction defects and arrhythmias in Duchenne muscular dystrophy. Am J Physiol Heart Circ Physiol.2020;318(6):H1436-H1440.

15. Chenard AA, Becane HM, Tertrain F, de Kermadec JM, Weiss YA. Ventricular arrhythmia in Duchenne muscular dystrophy: prevalence, significance and prognosis. Neuromuscul Disord.1993;3(3):201-206.

16. Juan-Mateu J, Gonzalez-Quereda L, Rodriguez MJ, et al. DMD Mutations in 576 Dystrophinopathy Families: A Step Forward in Genotype-Phenotype Correlations. PLoS One. 2015;10(8):e0135189.

17. Tandon A, Jefferies JL, Villa CR, et al. Dystrophin genotype-cardiac phenotype correlations in Duchenne and Becker muscular dystrophies using cardiac magnetic resonance imaging. Am J Cardiol.2015;115(7):967971.

18. Kaspar RW, Allen HD, Ray WC, et al. Analysis of dystrophin deletion mutations predicts age of cardiomyopathy onset in becker muscular dystrophy. Circ Cardiovasc Genet. 2009;2(6):544-551.

19. Yamamoto T, Awano H, Zhang Z, et al. Cardiac Dysfunction in Duchenne Muscular Dystrophy Is Less Frequent in Patients With Mutations in the Dystrophin Dp116 Coding Region Than in Other Regions. Circ Genom Precis Med. 2018;11(1):e001782.

20. Chiang DY, Allen HD, Kim JJ, et al. Relation of Cardiac Dysfunction to Rhythm Abnormalities in Patients With Duchenne or Becker Muscular Dystrophies. Am J Cardiol. 2016;117(8):1349-1354. 
21. Altekin RE, Yanikoglu A, Ucar M, Ermis C. Complete AV block and cardiac syncope in a patient with Duchenne muscular dystrophy. J Cardiol Cases. 2011;3(2):e68-e70.

22. Segawa K, Komaki H, Mori-Yoshimura M, et al. Cardiac conduction disturbances and aging in patients with Duchenne muscular dystrophy.Medicine (Baltimore). 2017;96(42):e8335.

23. Villa CR, Czosek RJ, Ahmed H, et al. Ambulatory Monitoring and Arrhythmic Outcomes in Pediatric and Adolescent Patients With Duchenne Muscular Dystrophy. J Am Heart Assoc. 2015;5(1).

24. Punnoose AR, Kaltman JR, Pastor W, McCarter R, He J, Spurney CF. Cardiac Disease Burden and Risk of Mortality in Hospitalized Muscular Dystrophy Patients. Pediatr Cardiol. 2016;37(7):1290-1296.

25. Epstein AE, DiMarco JP, Ellenbogen KA, et al. ACC/AHA/HRS 2008 Guidelines for Device-Based Therapy of Cardiac Rhythm Abnormalities: a report of the American College of Cardiology/American Heart Association Task Force on Practice Guidelines (Writing Committee to Revise the ACC/AHA/NASPE 2002 Guideline Update for Implantation of Cardiac Pacemakers and Antiarrhythmia Devices): developed in collaboration with the American Association for Thoracic Surgery and Society of Thoracic Surgeons. Circulation. 2008;117(21):e350-408.

26. Al-Khatib SM, Stevenson WG, Ackerman MJ, et al. 2017 AHA/ACC/HRS Guideline for Management of Patients With Ventricular Arrhythmias and the Prevention of Sudden Cardiac Death: Executive Summary: A Report of the American College of Cardiology/American Heart Association Task Force on Clinical Practice Guidelines and the Heart Rhythm Society. J Am Coll Cardiol. 2018;72(14):1677-1749.

27. Kadish A, Dyer A, Daubert JP, et al. Prophylactic defibrillator implantation in patients with nonischemic dilated cardiomyopathy. $N$ Engl J Med. 2004;350(21):2151-2158.

28. Dimas VV, Denfield SW, Friedman RA, et al. Frequency of cardiac death in children with idiopathic dilated cardiomyopathy. Am J Cardiol. 2009;104(11):1574-1577.

29. Pahl E, Sleeper LA, Canter CE, et al. Incidence of and risk factors for sudden cardiac death in children with dilated cardiomyopathy: a report from the Pediatric Cardiomyopathy Registry. J Am Coll Cardiol. 2012;59(6):607-615.

30. Corrado G, Lissoni A, Beretta S, et al. Prognostic value of electrocardiograms, ventricular late potentials, ventricular arrhythmias, and left ventricular systolic dysfunction in patients with Duchenne muscular dystrophy. Am J Cardiol. 2002;89(7):838-841.

31. Ponikowski P, Voors AA, Anker SD, et al. 2016 ESC Guidelines for the diagnosis and treatment of acute and chronic heart failure: The Task Force for the diagnosis and treatment of acute and chronic heart failure of the European Society of Cardiology (ESC). Developed with the special contribution of the Heart Failure Association (HFA) of the ESC.Eur J Heart Fail. 2016;18(8):891-975.

32. Yancy CW, Jessup M, Bozkurt B, et al. 2017 ACC/AHA/HFSA Focused Update of the 2013 ACCF/AHA Guideline for the Management of Heart Failure: A Report of the American College of Cardiology/American Heart Association Task Force on Clinical Practice Guidelines and the Heart Failure Society of America. $J$ Am Coll Cardiol.2017;70(6):776-803.

33. Mavrogeni SI, Markousis-Mavrogenis G, Papavasiliou A, Papadopoulos G, Kolovou G. Cardiac Involvement in Duchenne Muscular Dystrophy and Related Dystrophinopathies. Methods Mol Biol. 2018;1687:3142.

34. Raman SV, Hor KN, Mazur W, et al. Eplerenone for early cardiomyopathy in Duchenne muscular dystrophy: a randomised, double-blind, placebo-controlled trial. Lancet Neurol.2015;14(2):153-161.

35. Raman SV, Hor KN, Mazur W, et al. Stabilization of Early Duchenne Cardiomyopathy With Aldosterone Inhibition: Results of the Multicenter AIDMD Trial. J Am Heart Assoc. 2019;8(19):e013501. 
36. Raman SV, Hor KN, Mazur W, et al. Eplerenone for early cardiomyopathy in Duchenne muscular dystrophy: results of a two-year open-label extension trial. Orphanet J Rare Dis. 2017;12(1):39.

37. Tracy CM, Epstein AE, Darbar D, et al. 2012 ACCF/AHA/HRS focused update of the 2008 guidelines for device-based therapy of cardiac rhythm abnormalities: a report of the American College of Cardiology Foundation/American Heart Association Task Force on Practice Guidelines and the Heart Rhythm Society. [corrected]. Circulation.2012;126(14):1784-1800.

38. Bardy GH, Lee KL, Mark DB, et al. Amiodarone or an implantable cardioverter-defibrillator for congestive heart failure. $N$ Engl J Med. 2005;352(3):225-237.

39. Bourke JP, Guglieri M, Duboc D, Group EtWS. 238th ENMC International Workshop: Updating management recommendations of cardiac dystrophinopathyHoofddorp, The Netherlands, 30 November - 2 December 2018. Neuromuscul Disord. 2019;29(8):634-643.

40. Connuck DM, Sleeper LA, Colan SD, et al. Characteristics and outcomes of cardiomyopathy in children with Duchenne or Becker muscular dystrophy: a comparative study from the Pediatric Cardiomyopathy Registry. Am Heart J. 2008;155(6):998-1005.

41. McCulloch MA, Lal AK, Knecht K, et al. Implantable Cardioverter Defibrillator Use in Males with Duchenne Muscular Dystrophy and Severe Left Ventricular Dysfunction. Pediatr Cardiol.2020;41(5):925931.

42. Hiermeier UM, Baker C, Bourke JP. Exploring the acceptability of implantable defibrillators in patients with cardiac dystrophinopathy and carers. Open Heart. 2020;7(1):e001230. 